\title{
Paraneoplastic limbic encephalitis with late-onset magnetic resonance imaging findings: A case report
}

\author{
YOSHIYA TSUNODA ${ }^{1}$, TAKUMI KIWAMOTO ${ }^{1}$, SHINSUKE HOMMA ${ }^{1}$, YUUKI YABUUCHI ${ }^{1}$, \\ HARUNA KITAZAWA ${ }^{1}$, TOSHIHIRO SHIOZAWA ${ }^{1}$, KENSUKE NAKAZAWA ${ }^{1}$, TAKASHI HOSAKA ${ }^{2}$, \\ KAZUHIRO ISHII $^{2}$, AKIKO ISHII ${ }^{2}$, AKIRA TAMAOKA ${ }^{2}$ and NOBUYUKI HIZAWA ${ }^{1}$ \\ Departments of ${ }^{1}$ Pulmonary Medicine and ${ }^{2}$ Neurological Medicine, \\ Faculty of Medicine, University of Tsukuba, Tsukuba, Ibaraki 305-8575, Japan
}

Received February 8, 2017; Accepted April 11, 2017

DOI: $10.3892 / \mathrm{mco} .2017 .1311$

\begin{abstract}
Paraneoplastic limbic encephalitis (PLE), a paraneoplastic neurological syndrome (PNS), is a rare nervous system disorder that results from the indirect effects of tumors and is commonly associated with small-cell lung cancer (SCLC). Previous studies have reported that magnetic resonance imaging (MRI) may be useful for diagnosing LE. Temporal lobe abnormalities are observed using T2-weighted and fluid-attenuated inversion recovery sequences; however, such abnormalities are detected in only $60 \%$ of patients with PLE. The present study describes a case of PLE associated with SCLC, in which LE was observed using MRI 26 days after the first convulsive seizure. Although the serum and cerebrospinal fluid analyses for onconeural antibodies were negative, the findings of this case indicate that PLE should be considered in the differential diagnosis, and that repeated brain MRI may be more helpful for diagnosis, as the brain MRI findings may be normal during the early stages of PLE.
\end{abstract}

\section{Introduction}

Paraneoplastic neurological syndrome (PNS), a diverse group of disorders, results from the indirect effects of tumors on any part of the nervous system (1). Paraneoplastic limbic encephalitis (PLE), a syndrome phenotype of PNS, is a rare disorder characterized by subacute onset of short-term memory loss, seizures, irritability, depression and cognitive decline (1,2). Small-cell lung cancer (SCLC) and testicular germ-cell tumors are the most frequently observed neoplasms in patients with PLE $(2,3)$. PNS is often associated with

Correspondence to: Dr Takumi Kiwamoto, Department of Pulmonary Medicine, Faculty of Medicine, University of Tsukuba, 1-1-1 Tennodai, Tsukuba, Ibaraki 305-8575, Japan

E-mail: t-kiwamoto@md.tsukuba.ac.jp

Key words: paraneoplastic limbic encephalitis, paraneoplastic neurological syndromes, small-cell lung cancer, magnetic resonance imaging, onconeural antibody antibodies that bind to proteins that are shared between the nervous system and tumors (4). In particular, SCLC exhibits neuroendocrine characteristics and expresses a number of neuronal antigens (5). Magnetic resonance imaging (MRI) may assist in diagnosing LE, as the medial temporal lobes often exhibit increased signal on fluid-attenuated inversion recovery (FLAIR) images. Cerebral atrophy may be detected when there is persistent long-term cerebral inflammation (6). As these abnormalities have not been examined in a large series of patients, the typical timecourse of MRI findings remains unknown. The present study reports a case of PLE associated with SCLC in a 67-year-old patient. This patient had normal MRI findings when he experienced a convulsive seizure, whereas PLE was diagnosed by signals that appeared 26 days after symptom onset. The patient was successfully treated with combined-modality therapy.

\section{Case report}

A 67-year-old Japanese male patient was admitted to the Tsukuba University Hospital (Tsukuba, Japan) in May 2014 due to a generalized tonic-clonic seizure of unknown cause. The patient had a 50 pack-year smoking history. Eighteen days prior to admission, he experienced a generalized tonic-clonic seizure while driving and was transferred to the emergency department of a community hospital. Brain MRI (Fig. 1A), electrocardiogram (ECG), Holter ECG and echocardiogram revealed normal findings. Although cerebrospinal fluid (CSF) analysis revealed a mildly elevated protein level without malignant cells, the electroencephalogram was normal. Although the cause of the seizure was not determined by neurological examination, chest computed tomography (CT) revealed a mass in the hilum of the left lung and a nodule in the left lower lobe (Fig. 2). All the laboratory results were normal, apart from increased serum levels of pro-gastrin-releasing peptide (2,362.5 pg/ml; normal range, 0.0-80.7 pg/ml). Five days after seizure onset, the patient experienced a second convulsion and his cognitive dysfunction worsened. Although antiepileptic drug treatment was initiated, the patient's consciousness level gradually deteriorated and he was referred to the Tsukuba University Hospital for further assessment and treatment. Performing a neurological examination was difficult, as the 

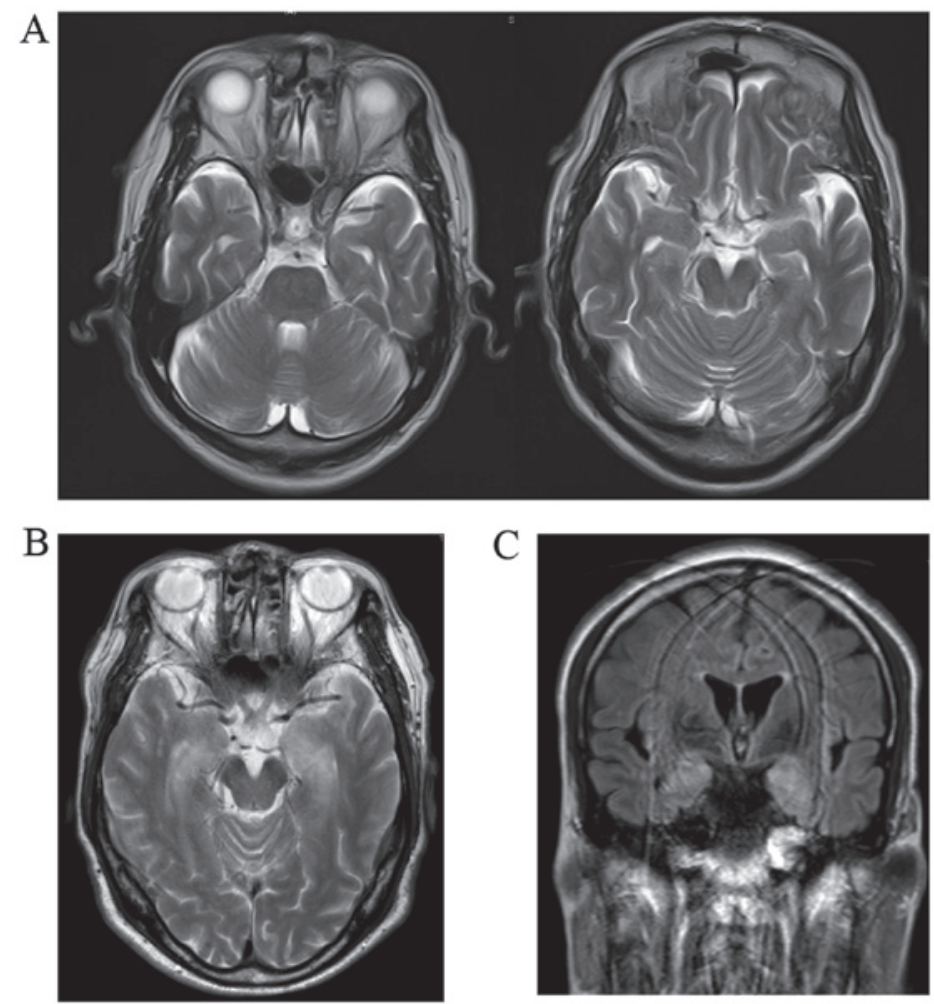

Figure 1. (A) The initial brain magnetic resonance imaging (MRI) was normal. (B and C) A repeat brain MRI 26 days after the initial scan identified bilateral limbic encephalitis in the medial temporal lobes. (B) T2-weighted axial section; (C) fluid-attenuated inversion recovery coronal section.
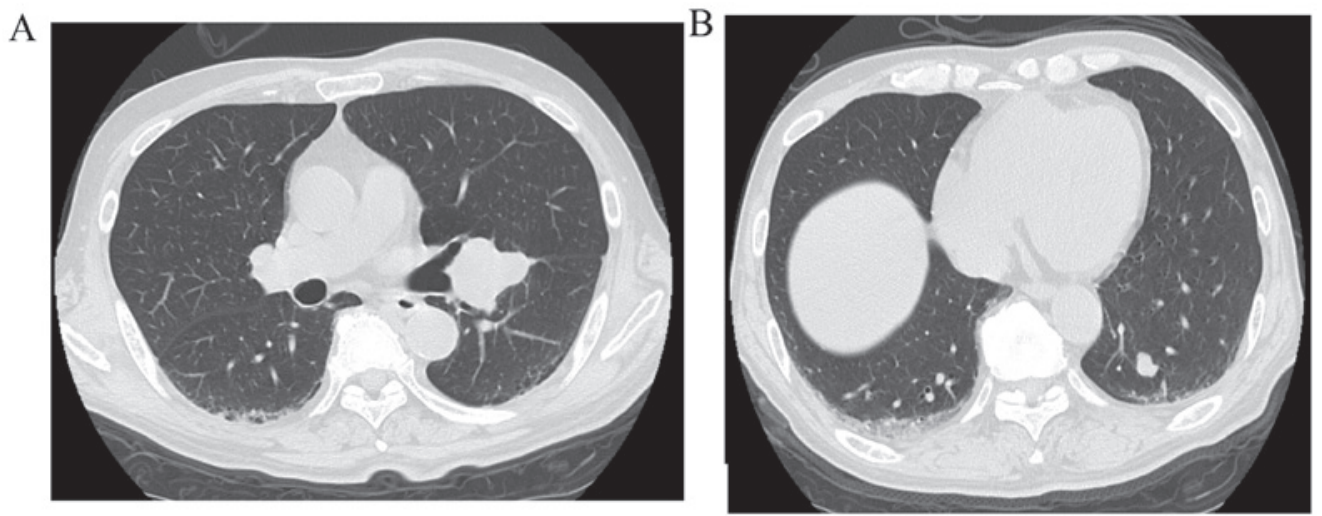

Figure 2. Computed tomography revealed (A) a mass in the left hilum of the lung and (B) a nodule in the left lower lobe.
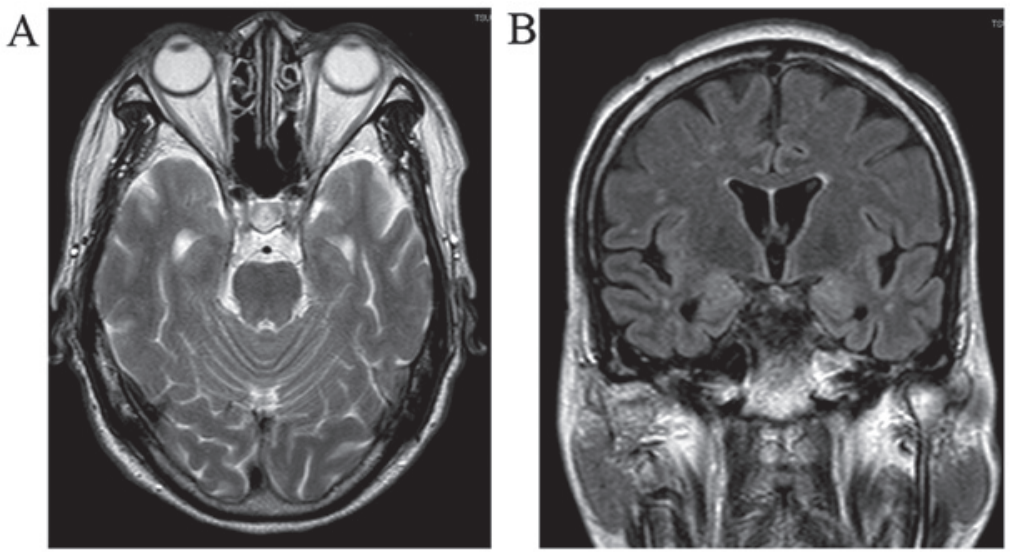

Figure 3. T2-weighted axial and fluid-attenuated inversion recovery coronal images on follow-up magnetic resonance imaging performed after 2 months of treatment revealed normal intensity; but atrophy of the medial temporal lobes. 
patient could not communicate at that time. The performance status (PS) was 4. Following admission, bronchoscopy was performed. Biopsy of transbronchial specimens from the mass in the left hilum revealed SCLC. Brain MRI was performed again 26 days after the first onset of the seizures. Although there were no metastatic lesions, high-intensity areas on T2-weighted and FLAIR images in the medial temporal lobes suggested a diagnosis of LE (Fig. 1B and C). Based on the available diagnostic criteria (7), the patient was diagnosed as having a definite PNS due to SCLC (limited disease). Serum and CSF analyses for onconeural antibodies [anti-Hu, anti-Yo, anti-Ri, anti-CV2, anti-amphiphysin, anti-Ma1, anti-Ma2 (PNS blot, Ravo Diagnostika GmbH, Freiburg, Germany) and anti-gephyrin] were negative. Due to the poor PS, curative radiotherapy ( $66 \mathrm{~Gy} / 33 \mathrm{Fr}$ ) for the primary tumor and metastatic lymph nodes was initiated. In conjunction with radiotherapy, high-dose intravenous corticosteroid therapy (1 g/day methylprednisolone for 3 days) was also initiated to relieve neurological symptoms. As the initial response to the steroid was unsatisfactory, intravenous pulse treatments were repeated once weekly for 3 weeks. Oral prednisolone $(0.5 \mathrm{mg} / \mathrm{kg} /$ day for 4 weeks) was subsequently administered. Following additional treatment, the patient's consciousness level gradually improved, and he was able to hold a simple conversation, but disorientation and memory disturbance remained. Two months after admission, the PS improved from 4 to 2, and chemotherapy with carboplatin (area under the curve $=5$, days $1-3$ for 4 weeks $)$ and etoposide $\left(100 \mathrm{mg} / \mathrm{m}^{2}\right.$, days 1-3 for 4 weeks) was initiated. After chemotherapy, a chest CT revealed partial clinical response in the primary tumor and lymph nodes. The neurological symptoms also disappeared, apart from a mild memory disturbance. T2-weighted and FLAIR images on follow-up brain MRI revealed normal intensity, but presence of atrophy in the medial temporal lobes (Fig. 3A and B). The patient was discharged from the hospital when he was ambulatory. At 14 months after the initial SCLC diagnosis, the primary tumor began to regrow, without encephalitis symptoms. The patient was successfully treated with second-line chemotherapy $\left(40 \mathrm{mg} / \mathrm{m}^{2}\right.$ amrubicin on days 1-3 for 4 weeks), and his disease was stable at the last follow-up in March 2017. Written informed consent was obtained from the patient for the publication of the case details and associated images.

\section{Discussion}

PNS is a neurological syndrome associated with cancer $(3,7)$. Over the last decades, several types of onconeural antibodies have been identified, suggesting that PNS is an immune-mediated syndrome. Although detecting onconeural antibodies is useful for diagnosing PNS, they cannot be detected in all patients with PNS. Consensus guidelines define classical PNS, wherein the tumor is diagnosed within 5 years, without onconeural antibodies, as 'definite PNS', since in patients with classical neurological syndromes, the tumor is almost always diagnosed within 5 years after the first PNS manifestation (7). In the literature, the frequency of PNS without causal antibodies is $12.5-18.3 \%(3,8)$. LE is classified as a typical syndrome that progresses with a subacute onset from several days to 12 weeks (7). Although $60 \%$ of patients with
PLE exhibit neurological symptoms prior to cancer diagnosis, $25-32 \%$ did not have detectable onconeural antibodies $(2,3)$. Neuropathological or neuroradiological evidence of the involvement of the limbic system is essential for diagnosing PLE without onconeural antibodies (7). The present study describes a case of PLE with SCLC. The diagnosis of LE was made on the basis of clinical and MRI findings.

The available data on the neuroradiological findings of PLE are currently limited. Urbach et al (9) studied serial MRI findings in 20 patients with LE and reported that typical MRI findings at the early stage of PLE are unilateral or bilateral temporomedial swelling and increased signal activity on T2-weighted FLAIR images. Hippocampal atrophy, with or without signal change, was also observed at later stages (9). Typical temporal lobe abnormalities on T2-weighted and FLAIR images were observed in $\sim 60 \%$ of patients with PLE $(2,9)$. In a proportion of PLE patients, MRI signals may emerge later than neurological symptoms, and the initial MRI may be negative (10). However, the proportion of patients with PLE with negative initial MRI findings remains unknown, and the present case provides important evidence regarding this possibility. ${ }^{18} \mathrm{~F}$-fluorodeoxyglucose positron emission tomography (FDG-PET) findings may precede the development of MRI findings and neurological symptoms. Several studies have suggested that FDG-PET is more sensitive compared with brain MRI in extratemporal regions, such as the cerebral cortex, brainstem and cerebellum (10-12). Unfortunately, FDG-PET was not performed in our case. When available, FDG-PET may contribute to early diagnosis of PLE.

Although there is currently no well-established treatment for PLE, corticosteroids are the treatment most frequently used, followed by high-dose immunoglobulins, plasma exchange and immunosuppressive drugs (2,3). For the treatment of tumors, chemotherapy is the most frequent option $(2,3)$. Gultekin et al reported the neurological outcome in 34 patients with PLE (2). Improvement was observed in 11 of 14 patients receiving tumor treatment alone, and in 4 of 14 patients receiving tumor treatment plus immunomodulatory therapy (2). Improvement was not observed in patients receiving immunomodulatory therapy alone (2). A recent study suggested that patients with PNS having onconeural antibodies targeting neuronal cell surface antigens (e.g., anti-VGKC and anti-NMDAR antibodies) exhibit a better treatment response compared with patients with PNS having onconeural antibodies targeting intracellular antigens (e.g., anti-Hu and anti-Ma antibodies) (13). Although in the present case no onconeural antibodies were detected, a satisfactory treatment response was observed after a repeated course of high-dose corticosteroid and anticancer therapies.

In conclusion, the present study reports a rare case of a patient in whom PLE was diagnosed by MRI signals that were subsequently observed in the medial temporal lobes 26 days after the first seizure onset. Our case indicates that PLE was not excluded from the differential diagnosis, even when the first brain MRI revealed normal findings; thus, MRI should be repeated if PLE is suspected.

\section{References}

1. Höftberger R, Rosenfeld MR and Dalmau J: Update on neurological paraneoplastic syndromes. Curr Opin Oncol 27: 489-495, 2015 
2. Gultekin SH, Rosenfeld MR, Voltz R, Eichen J, Posner JB and Dalmau J: Paraneoplastic limbic encephalitis: Neurological symptoms, immunological findings and tumour association in 50 patients. Brain 123: 1481-1494, 2000

3. Giometto B, Grisold W, Vitaliani R, Graus F, Honnorat J and Bertolini G; PNS Euronetwork: Paraneoplastic neurologic syndrome in the PNS Euronetwork database: A European study from 20 centers. Arch Neurol 67: 330-335, 2010.

4. Gozzard P and Maddison P: Which antibody and which cancer in which paraneoplastic syndromes? Pract Neurol 10: 260-270, 2010.

5. Benatar M, Blaes F, Johnston I, Wilson K, Vincent A, Beeson D and Lang B: Presynaptic neuronal antigens expressed by a small cell lung carcinoma cell line. J Neuroimmunol 113: 153-162, 2001.

6. Mason WP, Graus F, Lang B, Honnorat J, Delattre JY, Valldeoriola F, Antoine JC, Rosenblum MK, Rosenfeld MR, Newsom-Davis J, et al: Small-cell lung cancer, paraneoplastic cerebellar degeneration and the Lambert-Eaton myasthenic syndrome. Brain 120: 1279-1300, 1997.

7. Greenlee JE: Recommended diagnostic criteria for paraneoplastic neurological syndromes. J Neurol Neurosurg Psychiatry 75: 1090, 2004

8. Gozzard P, Woodhall M, Chapman C, Nibber A, Waters P, Vincent A, Lang B and Maddison P: Paraneoplastic neurologic disorders in small cell lung carcinoma: A prospective study. Neurology 85: 235-239, 2015.

9. Urbach H, Soeder BM, Jeub M, Klockgether T, Meyer B and Bien CG: Serial MRI of limbic encephalitis. Neuroradiology 48: 380-386, 2006.
10. Serafini A, Lukas RV, VanHaerents S, Warnke P, Tao JX, Rose S and Wu S: Paraneoplastic epilepsy. Epilepsy Behav 61: 51-58, 2016.

11. Ances BM, Vitaliani R, Taylor RA, Liebeskind DS, Voloschin A, Houghton DJ, Galetta SL, Dichter M, Alavi A, Rosenfeld MR and Dalmau J: Treatment-responsive limbic encephalitis identified by neuropil antibodies: MRI and PET correlates. Brain 128: 1764-1777, 2005

12. Scheid R, Lincke T, Voltz R, von Cramon DY and Sabri O: Serial 18F-fluoro-2-deoxy-D-glucose positron emission tomography and magnetic resonance imaging of paraneoplastic limbic encephalitis. Arch Neurol 61: 1785-1789, 2004.

13. Dalmau J and Rosenfeld MR: Paraneoplastic syndromes of the CNS. Lancet Neurol 7: 327-340, 2008. 